\title{
Comments on: Comparing and selecting spatial predictors using local criteria
}

\author{
M. D. Ruiz-Medina
}

Published online: 29 November 2014

(C) Sociedad de Estadística e Investigación Operativa 2014

Local criteria based on validation data to select a predictor at each spatial location of a domain $D \subset \mathbb{R}^{d}$ are proposed in a non-parametric setting. The resulting combination of spatial predictors is referred as locally selected predictor (LSP). Classical and more recently formulated spatial predictors can be considered as candidates in the associated local selection problem. Comparison of different LSPs and globally selected predictors (GSPs) is performed in a simulation study. LSP is applied to a real Big Data problem in relation to atmospheric $\mathrm{CO}_{2}$ measurements.

The methodology proposed, based on combination of spatial predictors, offers a flexible framework for optimal spatial prediction in the statistical analysis of data displaying spatial heterogeneities. The non-parametric framework adopted allows its application without considering any specific model assumptions on the latent process $Y$. Even in the case of considering a state space spatial context for definition of the spatial dynamics of $Y$, equation (18) can be fitted to this framework by projection into a suitable functional basis. Note that dimension reduction techniques, based on numerical projection, are usually applied for processing high-dimensional data.

On the other hand, in the simulation study developed, the authors consider a negative exponential covariance function to calibrate the spatial dependence structure of $S(\mathbf{u})^{\prime} \boldsymbol{\eta}$, given from $\mathbf{K}=\operatorname{cov}(\boldsymbol{\eta})$, involved in the definition of latent process $Y$ in equation (18). This model is spatially homogeneous and isotropic. Perhaps, a spatially heterogeneous covariance model should be tested, keeping in mind that, in addition, the spatial observation noise $\varepsilon$ is allowed to have a spatially heterogeneous variance in Section 2.1.

This comment refers to the invited paper available at doi:10.1007/s11749-014-0415-1.

M. D. Ruiz-Medina $(\varangle)$

Universidad de Granada, Granada, Spain

e-mail:mruiz@ugr.es 
In the proof of equation (10) in Appendix 1, for obtaining equation (27) from equation (26), after taking expectation at both sides of equation (26), I think the authors apply the global independence of process $\varepsilon$ with respect to process $Y$, assumed in Section 2.1, since the $\widehat{k}$ th spatial predictor considered depends on $Z\left(s_{j}^{\mathrm{tm}}\right), j=$ $1, \ldots, n$, which also depends on $Y\left(s_{j}^{\mathrm{tm}}\right), j=1, \ldots, n$, i.e., the $\widehat{k}$ th spatial predictor is computed from the training data.

The following considerations could lead to an improvement of the proposed locally selected spatial predictor:

In the context of Big Data to reduce dimensionality of the LSP, spatial sampling design techniques could be applied in the selection of a training sample from a set of candidates, for computation of the spatial predictors, considering a suitable loss function, perhaps, in a non-parametric framework.

In practice, when observable locations are partitioned into training and validation locations, it could be possible to run different options of randomly selected training and validation samples to evaluate LSP in equation (7). Specifically, for each $k=$ $1, \ldots, K$, and for $i=1, \ldots, N, \operatorname{LSVE}^{(k)}\left(\mathbf{u}_{i}, H\right)$ should be given by the average over the runs of the squared validation errors obtained for each randomly selected training and validation samples, in the spirit of the cross-validation methodology. Hence, $\widehat{\mathbf{Y}}^{L S P}$ is evaluated taking at each spatial location the minimum over the averaged squared validation errors computed for the spatial predictors tested.

The dependence of the proposed locally selected predictor on the set $H$ should be taken into account in the application of this methodology. Some criteria for solving this model selection problem could improve the application in practice of the LSP methodology. 